Journal for

ImmunoTherapy of Cancer

\title{
Tracking the tail
}

\author{
Alex Friedlaender, ${ }^{1}$ Stephen V Liu (i) , ${ }^{2}$ Alfredo Addeo ${ }^{1}$
}

To cite: Friedlaender A, Liu SV, Addeo A. Tracking the tail. Journal for ImmunoTherapy of Cancer 2020;8:e000971. doi:10.1136/jitc-2020-000971

Accepted 06 August 2020

Check for updates

(C) Author(s) (or their employer(s)) 2020. Re-use permitted under CC BY-NC. No commercial re-use. See rights and permissions. Published by BMJ.

${ }^{1}$ Oncology, Geneva University Hospitals, Geneva, Switzerland ${ }^{2}$ Medical Oncology, Georgetown University, Washington, District of Columbia, USA

Correspondence to

Dr Alfredo Addeo;

alfredo.addeo@hcuge.ch

\section{ABSTRACT}

Immune-checkpoint inhibitors have deeply changed the therapeutic landscape of advanced non-small cell lung cancer without actionable genomic alterations. Immune-checkpoint inhibitors have become standard front-line therapy, especially among patients with tumours expressing high levels of programmed death ligand-1; yet, many patients do not respond to therapy. This has led to the adoption of front-line combination therapies, administering programmed death-1 inhibitors concomitantly either with other checkpoint inhibitors, chemotherapy or both. Today's approved standard of care includes options with chemoimmunotherapy or dual checkpoint blockade, but each combination has only been compared to chemotherapy alone and no head-to-head trials exist. In cross-trial comparisons, combinations trials appear to show numerically superior responses to single-agent checkpoint inhibitors but the question is whether they ultimately offer a survival advantage. In this manuscript, we summarize and analyse all currently available front-line immune-checkpoint inhibitor trials in non-small cell lung cancer, whether as monotherapy or in combination with chemotherapy, second immunotherapy agents or both. Should standards of care change given the current data? While we ponder this question, we illustrate current data and conclude that the answer lies in tracking the tail of the survival curves.

Immune-checkpoint inhibitors (ICIs) have deeply changed the therapeutic landscape of advanced non-small cell lung cancer (NSCLC) without actionable genomic alterations. Targeting the programmed death-1/ programmed death ligand-1 (PD-1/PD-L1) axis has emerged as the standard of care, initially in second-line, then first-line therapy, with potential long-term survival in a subset of patients. ${ }^{1}$

Following positive results in KEYNOTE-001, ${ }^{1}$ pembrolizumab, an anti-PD-1 checkpoint inhibitor, was Food and Drug Administration (FDA)-approved as second-line monotherapy for NSCLC. Subsequently, in KEYNOTE-010, pembrolizumab confirmed an overall survival (OS) benefit, compared with second-line docetaxel in patients with tumors expressing PD-L1 in $\geq 1 \%$ of cells. ${ }^{2}$ Further randomized phase III trials with both atezolizumab, an anti-PD-L1 antibody, and nivolumab, an anti-PD-1 antibody, yielded similar results compared with docetaxel in the second-line, this time including patients whose tumors did not express PD-L1. ${ }^{34}$

Subsequently, KEYNOTE-024 and KEYNOTE-042 compared front-line pembrolizumab to platinum-based chemotherapy in tumors with PD-L1 $\geq 50 \%$ and $1 \%$, respectively. ${ }^{56}$ Both studies showed an OS advantage which led to FDA approval of pembrolizumab in both patients population. ${ }^{7}$ Followed this, an international panel of thoracic oncologists published a position paper to share their concern about the approval of pembrolizumab in patients with NSCLC with PD-L1 $>1 \% .{ }^{8}$ The authors raised a few points: first of all in the KEYNOTE-042 nearly half $(46.6 \%)$ of the patients enrolled had a PD-L1 $\geq 50 \%$, which represented a potential bias for the over-performing efficacy of pembrolizumab in the intention to treat population. Second in the predefined OS analysis by PD-L1 expression, a survival benefit from pembrolizumab was not seen in the PD-L1 1\%-49\% subgroup (median OS 13.4 vs 12.1 months, HR 0.92 , $95 \%$ CI 0.77 to 1.11 ). This suggests that the observed benefit with pembrolizumab has been largely driven by the "high PD-L1 expression" group, in which the HR for OS benefit mirrors that from a similar population in the KEYNOTE024 trial (0.69 and 0.63 , respectively). They concluded by stating that pembrolizumab monotherapy might not be the best treatment for patients with tumor PD-L1 of 1\%-49\% and this strategy could expose patients at risk of fast progression.

Similar findings were echoed by atezolizumab in IMpower $110,{ }^{9}$ where a benefit in terms of OS was again driven by high PD-L1 expressors. ${ }^{9}$ In this trial, nearly $40 \%$ of the patients in both arms had a PD-L1 $\geq 50 \%$. Unlike other trials in which the importance of high PD-L1 expression was known, the Checkmate 026 trial set a $5 \%$ cut-off for PD-L1 and did not stratify for PD-L1 $\geq 50 \%$. The trial aimed to prove that nivolumab could improve progression-free survival (PFS) compared with standard platinum-based chemotherapy in that population. It failed to meet its primary endpoint. ${ }^{10}$ It is nonetheless worth mentioning that there was a clear imbalance 
Table 1 Selection of phase III combination chemotherapy-checkpoint inhibitor trials

\begin{tabular}{llllll}
\hline Trial name & Histology & Exp arm & mPFS & mOS & HR (95\% Cl) \\
\hline IMpower130 & NSCC & Atezo-CnP & 7.0 & 18.6 & $0.79(0.64$ to 0.98) \\
IMpower131 & SCC & Atezo-CnP & 6.5 & 14.6 & $0.88(0.73$ to 1.05) \\
IMpower132 & NSCC & Atezo-PP & 7.6 & 18.1 & $0.81(0.64$ to 1.03) \\
IMpower150 & NSCC & Atezo-B-CP & 8.3 & 19.2 & $0.78(0.64$ to 0.96) \\
Keynote189 & NSCC & Pembro-PP & 8.8 & 22.0 & $0.56(0.45$ to 0.70) \\
Keynote407 & SCC & Pembro-CP or CnP & 6.4 & 17.1 & $0.71(0.58$ to 0.88) \\
\hline
\end{tabular}

Atezo, atezolizumab; B, bevacizumab; CnP, carboplatin nab-paclitaxel; CP, carboplatin paclitaxel; Exp arm, experimental arm; mOS, median overall survival; mPFS, median progression-free survival; NSCC, non-squamous cell carcinoma; Pembro, pembrolizumab; PP, platinum pemetrexed; SCC, squamous cell carcinoma.

among high PD-L1 expressors with $41 \%$ in the nivolumab arm and $60 \%$ in the chemotherapy arm.

However, many patients do not respond, leading to the adoption of front-line chemotherapy-ICI combinations across PD-L1 expression subtypes ${ }^{11}$ (table 1).

Alternate strategies have explored combined PD-1 and CTLA-4 checkpoint blockade with conflicting results. Rizvi and colleagues ${ }^{12}$ recently reported findings from the Mystic trial. 1118 patients with previously untreated metastatic NSCLC without EGFR or $A L K$ gene alterations were randomized (1:1:1) to receive platinumbased doublet chemotherapy, single-agent durvalumab (anti-PD-L1), or durvalumab with tremelimumab (antiCTLA-4) combination therapy. It should be noted that KRAS alterations are not an exclusion factor, as these appear not to influence response to checkpoint inhibitors. ${ }^{1314}$ The primary endpoints of the study were assessed in the 488 patients with PD-L1 expression in $25 \%$ of tumor cells or more and included OS for durvalumab versus platinum-based doublet chemotherapy, and OS and PFS for the durvalumab and tremelimumab combination versus chemotherapy. The study did not meet its primary endpoints. The median OS in patients with PD-L1 $\geq 25 \%$ was 16.3 months for patients treated with durvalumab, compared with 12.9 months with platinum-based doublet chemotherapy, without a statistically significant difference. The median OS for the durvalumab and tremelimumab combination was 11.9 months, which was lower than both the durvalumab and the chemotherapy arms. The median PFS for durvalumab and tremelimumab was quite disappointing, at 3.9 months, whereas the PFS with chemotherapy was 5.4 months.

The second coprimary endpoint of Checkmate 227, OS in patients with tumors with PD-L1 $\geq 1 \%$, compared nivolumab plus ipilimumab to chemotherapy. ${ }^{15}$ The median OS was 17.1 months (95\% CI 15.0 to 20.1) with nivolumab plus ipilimumab and 14.9 months $(95 \%$ CI 12.7 to 16.7$)$ with chemotherapy $(\mathrm{p}=0.007)$. OS rates at 1 and 2 years were $62.6 \%$ and $40.0 \%$, respectively, with nivolumab plus ipilimumab, compared with $56.2 \%$ and $32.8 \%$ with chemotherapy. The HR for death of 0.79 (97.72\% CI 0.65 to 0.96 ) should be interpreted in the context of the shape of the curves: a transient initial survival benefit with chemotherapy, followed by a significant long-term benefit with nivolumab plus ipilimumab. Response rate (RR) was $35.9 \%$ (95\% CI 31.1 to 40.8 ) with nivolumab plus ipilimumab versus $30.0 \%$ (95\% CI 25.5 to 34.7$)$ with chemotherapy. Median duration of response among PD-L1 positive patients was 23.2 months (95\% CI 15.2 to 32.2$)$ with nivolumab plus ipilimumab and 6.2 months (95\% CI 5.6 to 7.4 ) with chemotherapy. More patients had an ongoing response with nivolumab plus ipilimumab than chemotherapy $(64.2 \%$ vs $27.9 \%$ at 1 year, $49.5 \%$ vs $11.0 \%$ at 2 years). Recently, the 3-year OS update was released at the American Society of Clinical Oncology 2020 Meeting, confirming durable benefit for the double immunotherapy blockade in the PD-L1 $>1 \%$ cohort. $^{16}$ The median OS was 17.1 months with nivolumab plus ipilimumab compared with 14.9 months with chemotherapy, with a HR of 0.79 . OS rates at 3 years were $33 \%$ with nivolumab plus ipilimumab, compared with $22 \%$ with chemotherapy.

Nivolumab plus ipilimumab was also compared with chemotherapy in a prespecified descriptive analysis in patients with PD-L1 $<1 \%$, as well as in the entire population. Median OS was again longer with nivolumab plus ipilimumab (17.2 months; 95\% CI 12.8 to 22.0 ) than chemotherapy (12.2 months; $95 \%$ CI 9.2 to 14.3 ), with a HR for death of $0.62(95 \%$ CI 0.48 to 0.78$)$ in that subgroup 2-year OS was $40.4 \%$ for nivolumab plus ipilimumab and $23.0 \%$ for chemotherapy. In all trial patients, duration of response to nivolumab plus ipilimumab was 19.6 months and 5.8 months with chemotherapy. The 3-year OS update confirmed durable benefit for the double immunotherapy blockade even in this exploratory subgroup. ${ }^{16}$ The median OS was 17.2 months with nivolumab plus ipilimumab versus 12.2 months with chemotherapy with a HR of 0.64 . OS rates at 3 years were $34 \%$ with nivolumab plus ipilimumab, compared with $15 \%$ with chemotherapy.

The ongoing RR in the Checkmate 227 trial was $49 \%$ with nivolumab plus ipilimumab, but only $11 \%$ with chemotherapy at the 24-month analysis. Nevertheless, the OS was longer with nivolumab plus ipilimumab in all 
trial patients, including those with PD-L1 $<1 \%$, a population for whom anti-PD-1 monotherapy is not an effective option. Although the relative benefit of nivolumab plus ipilimumab compared with chemotherapy was numerically greater in patients with tumors expressing PD-L1 $<1 \%$ than in those with PD-L1 $>1 \%$, it was likely due to performance variations in control arms. Median duration and rates of 1-year and 2-year OS with nivolumab plus ipilimumab were nearly identical in both PD-L1 subgroups, but chemotherapy underperformed in the PD-L1 $<1 \%$ group.

The Checkmate 9LA trial (NCT03215706) was designed in order to provide rapid disease control while building on the durable OS benefit observed with the combination of nivolumab and ipilimumab, the. In this phase III trial, two cycles of standard platinum-based chemotherapy were added upfront to the dual immune blockade, compared with standard platinum-based chemotherapy in first-line stage IV NSCLC. The primary endpoint was OS, and secondary endpoints included PFS, objective response rate (ORR), and efficacy by PD-L1 expression. The combination of chemotherapy, nivolumab and ipilimumab was previously explored and proven to be safe in part 2 of the Checkmate 568 trial. $^{17}$

During a minimum follow-up of 12.7 months, nivolumab and ipilimumab with chemotherapy resulted in prolonged OS, with a median of 15.6 months compared with 10.9 months in patients receiving chemotherapy alone, with a HR of 0.66 . The 1 -year OS was $63 \%$ in the combination group compared with $47 \%$ in the chemotherapyonly group. The benefit was seen regardless of PD-L1 positivity, histology and multiple subgroups. There was, however, reduced efficacy among patients older than 75 and never smokers. Median PFS was also improved in the combination arm, at 6.7 months compared with 5.0 months with chemotherapy alone (HR, 0.68; 95\% CI 0.57 to 0.82 ). The 1 -year PFS was $33 \%$ and $18 \%$ in the combination and chemotherapy arms, respectively. The ORR was $38 \%$ with the combination compared with $25 \%$ with chemotherapy alone. The median duration of response was 11.3 months with nivolumab and ipilimumab plus chemotherapy compared with 5.6 months with chemotherapy.

The median OS, PFS and early landmark OS are important when evaluating efficacy, but the critical benchmark for success with immunotherapy is long-term survival. Improved 5 and even 10-year survival rates are new measures of success. The tail of the Kaplan-Meier curve captures our imagination. A subset of heavily pretreated patients from phase I ICI trials remains alive years later. ${ }^{1}$ Chemotherapy-ICI or ICI-ICI each have relative merits but how we gage success will be long-term OS. Unfortunately, there is no clear surrogate, no shortcut to those results. Chemotherapy improves RR and short-term PFS, but will that translate to long-term survival? Could cytotoxic chemotherapy ultimately hamper formation of immune-memory, improving short-term results but mortgaging long-term OS? There is no clear answer to

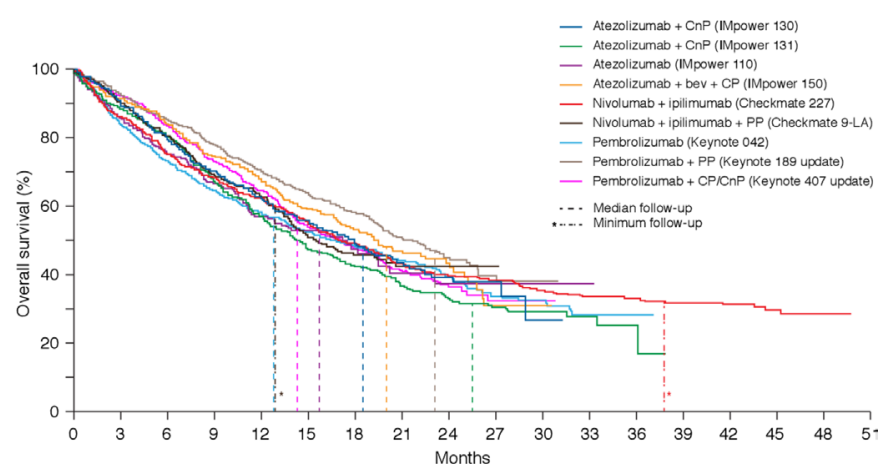

Figure 1 Overall survival curves from current front-line checkpoint-inhibitor trials in non small-cell lung cancer. CnP, carboplatin nab-paclitaxel; CP, carboplatin paclitaxel; PP, platinum pemetrexed.

these pressing questions for now, but perhaps a longer follow-up will provide much needed clarity.

We summarize current data in a visual representation of Kaplan-Meier curves of first-line options for fit patients, ${ }^{18}$ including median follow-up as a reminder that current tails are not yet mature (figure 1 ). We conclude by pondering whether the standard of care has changed after Checkmate 227 and Checkmate 9LA. In our opinion, it has not, at least not yet. We need time to evaluate long-term efficacy of each strategy. It is unlikely that one size fits all, as unique patients will need individualized approaches, so it remains crucial to develop biomarkers. ${ }^{120}$ There are now several first-line options and we will develop our own practice patterns, but we must reassess as more mature data emerge.

We will place different values on the advantages of each strategy, but for us, tracking the tail will guide us to the answer.

\section{Twitter Stephen V Liu @StephenVLiu}

Contributors All the authors have equally contributed to the manuscript.

Funding The authors have not declared a specific grant for this research from any funding agency in the public, commercial or not-for-profit sectors.

Competing interests AA has received compensation from Bristol-Myers Squibb, AstraZeneca, Merck Sharpe \& Dohme, Takeda, Pfizer, Roche and Boehringer Ingelheim for participating on advisory boards. SVL Research: Ignyta, Genentech, Pfizer, Threshold, Clovis, Corvus, Esanex, Bayer, OncoMed, Merck, Lycera, AZ, Molecular Partners, Rain Therapeutics; Advisory boards: Ignyta, Genentech, Pfizer, Takeda, Celgene, Lilly, Taiho, BMS, AZ, Regeneron, Merck. S.P. Chawla: Honoraria/ research/Advisory boards: Amgen, Roche, GSK, Threshold Pharmaceuticals, CytRx Corporation, Ignyta, Immune Design, TRACON Pharma, Karyopharm Therapeutics, Sarc, Janssen. AF has received compensation from Roche, Pfizer, Astellas and Bristol-Myers Squibb for service as a consultant.

\section{Patient consent for publication Not required.}

Provenance and peer review Not commissioned; externally peer reviewed.

Open access This is an open access article distributed in accordance with the Creative Commons Attribution Non Commercial (CC BY-NC 4.0) license, which permits others to distribute, remix, adapt, build upon this work non-commercially, and license their derivative works on different terms, provided the original work is properly cited, appropriate credit is given, any changes made indicated, and the use is non-commercial. See http://creativecommons.org/licenses/by-nc/4.0/.

\section{ORCID iD}

Stephen V Liu http://orcid.org/0000-0002-4852-3914 


\section{REFERENCES}

1 Leighl NB, Hellmann MD, Hui R, et al. Pembrolizumab in patients with advanced non-small-cell lung cancer (KEYNOTE-001): 3-year results from an open-label, phase 1 study. Lancet Respir Med 2019;7:347-57.

2 Herbst R, Garon E, Kim D-W, et al. OA03.07 KEYNOTE-010: durable clinical benefit in patients with previously treated, PD-L1-Expressing NSCLC who completed pembrolizumab. Journal of Thoracic Oncology 2017;12:S254-5.

3 Rittmeyer A, Barlesi F, Waterkamp D, et al. Atezolizumab versus docetaxel in patients with previously treated non-small-cell lung cancer (oak): a phase 3, open-label, multicentre randomised controlled trial. Lancet 2017;389:255-65.

4 Borghaei H, Paz-Ares L, Horn L, et al. Nivolumab versus docetaxel in advanced Nonsquamous non-small-cell lung cancer. N Engl J Med 2015;373:1627-39.

5 Mok TSK, Wu Y-L, Kudaba I, et al. Pembrolizumab versus chemotherapy for previously untreated, PD-L1-expressing, locally advanced or metastatic non-small-cell lung cancer (KEYNOTE-042): a randomised, open-label, controlled, phase 3 trial. Lancet 2019;393:1819-30.

6 Reck M, Rodríguez-Abreu D, Robinson AG, et al. Updated Analysis of KEYNOTE-024: Pembrolizumab Versus Platinum-Based Chemotherapy for Advanced Non-Small-Cell Lung Cancer With PD-L1 Tumor Proportion Score of $50 \%$ or Greater. J Clin Oncol 2019;37:537-46.

7 FDA. Approval pembro in NSCLC patients with PD-L1>1\%, 2019. Available: https://wwwfdagov/drugs/fda-expands-pembrolizumabindication-first-line-treatment-nsclc-tps-1

8 Mountzios G, Remon J, Novello S, et al. Position of an international panel of lung cancer experts on the decision for expansion of approval for pembrolizumab in advanced non-small-cell lung cancer with a PD-L1 expression level of $\geq 1 \%$ by the USA Food and Drug Administration. Ann Oncol 2019;30:1686-8.

9 Spigel D. Impower110: interim overall survival (OS) analysis of a phase III study of atezolizumab (atezo) vs platinum-based chemotherapy (chemo) as first-line $(1 \mathrm{~L})$ treatment $(t x)$ in $P D$ L1-selected NSCLC. Abstract LBA78. Barcelona, Spain: ESMO Congress 2019, 2019.
10 Carbone DP, Reck M, Paz-Ares L, et al. First-Line nivolumab in stage IV or recurrent non-small-cell lung cancer. $N$ Engl J Med 2017;376:2415-26.

11 Addeo A, Banna GL, Metro G, et al. Chemotherapy in combination with immune checkpoint inhibitors for the first-line treatment of patients with advanced non-small cell lung cancer: a systematic review and Literature-Based meta-analysis. Front Oncol 2019;9:264.

12 Rizvi NA, Cho BC, Reinmuth N, et al. Durvalumab with or without tremelimumab vs standard chemotherapy in first-line treatment of metastatic non-small cell lung cancer: the MYSTIC phase 3 randomized clinical trial. JAMA Oncol 2020. [Epub ahead of print: 09 Apr 2020].

13 Friedlaender A, Drilon A, Weiss GJ, et al. Kras as a druggable target in NSCLC: rising like a Phoenix after decades of development failures. Cancer Treat Rev 2020;85:101978.

14 Torralvo J, Friedlaender A, Achard V, et al. The activity of immune checkpoint inhibition in KRAS mutated non-small cell lung cancer: a single centre experience. Cancer Genom Proteom 2019;16:577-82.

15 Hellmann MD, Paz-Ares L, Bernabe Caro R, et al. Nivolumab plus ipilimumab in advanced non-small-cell lung cancer. $N$ Engl $J$ Med 2019;381:2020-31.

16 Ramalingam SS, Ciuleanu TE, Pluzanski A. Nivolumab+ ipilimumab versus platinum-doublet chemotherapy as first-line treatment for advanced non-small cell lung cancer:Three-year update from CheckMate 227 Part 1. Am J Clin Oncol 2020.

17 Gainor JF, Schneider JG, Gutierrez M, et al. Nivolumab (NIVO) plus ipilimumab (IPI) with two cycles of chemotherapy (chemo) in first-line metastatic non-small cell lung cancer (NSCLC): CheckMate 568 Part 2. JCO 2020;38:9560.

18 Friedlaender A, Banna GL, Buffoni L, et al. Poor-Performance status assessment of patients with non-small cell lung cancer remains vague and blurred in the immunotherapy era. Curr Oncol Rep 2019;21:107.

19 Addeo A, Banna GL, Weiss GJ. Tumor mutation Burden-From hopes to doubts. JAMA Oncol 2019;5:934-5.

20 Friedlaender A, Bauml J, Banna GL, et al. Identifying successful biomarkers for patients with non-small-cell lung cancer. Lung Cancer Manag 2019;8:Lmt17. 